\title{
The Cerebellum Plays a Role in Cognitive, but Not Affective, Theory of Mind: A Voxel-Based Lesion Mapping Study
}

pierre-aurélien beuriat ( $\nabla$ pierre-aurelien.beuriat@neurochirurgie.fr )

Northwestern University

\section{Shira Cohen-Zimerman}

Northwestern University

Gretchen Smith

Shirley Ryan AbilityLab

Frank Krueger

George Mason University

\section{Barry Gordon}

Johns Hopkins University

Jordan Grafman

Northwestern University

\section{Research Article}

Keywords: mentalizing, cerebellum, social cognition, white matter tract, brain injury

Posted Date: July 28th, 2021

DOl: https://doi.org/10.21203/rs.3.rs-735720/v1

License: (9) (i) This work is licensed under a Creative Commons Attribution 4.0 International License. Read Full License 


\section{Abstract}

Introduction: Theory of Mind (ToM) is a social-cognitive skill that allows the understanding of the intentions, beliefs, and desires of others. There is a distinction between affective and cognitive ToM, with evidence showing that these processes rely on partially distinct neural networks. The role of the cerebellum in social cognition has only been rarely explored. In this study, we tested whether the cerebellum is necessary for cognitive and affective ToM performance.

Material and methods: We investigated adults with traumatic brain injury $(n=193)$ and healthy controls $(n=52)$ using voxel-based lesion-symptom mapping (VLSM) and by measuring the impact on functional connectivity.

Results: First, we observed that damage to the cerebellum affected Cognitive but not Affective ToM processing. Further, we found a lateralization effect for the role of the cerebellum in cognitive ToM with participants with left cerebellar injury performing worse than those with right cerebellar injury. Both VLSM and standard statistical analysis provided evidence that left cerebellar Crus I and lobule VI contributed to ToM processing. Lastly, we found that disconnection of the left thalamic projection and the left frontostriatal fasciculus was associated with poor cognitive ToM performance.

Conclusions: Our study is the first to reveal direct causal neuropsychological evidence for a role of the cerebellum in cognitive, but not in affective, ToM, processing. It reinforces the idea that social cognition relies on a complex network functionally connected through white matter pathways that include the cerebellum. It supports evidence that the neural networks underpinning cognitive and affective ToM can be differentiated.

\section{Introduction}

Theory of Mind (ToM) is a complex social-cognitive skill. Studies investigating the neural underpinnings of ToM, emphasized the role of cortical regions ${ }^{1}$. ToM abilities seem to mainly rely on the default/mentalizing network. Moreover, studies suggest an overall cortical laterality effect with ToM being linked to the right hemisphere ${ }^{2}$. A distinction has been made between two different ToM processes: affective ToM (i.e., the ability to infer others' emotional states and feelings) and cognitive ToM (i.e., the ability to infer others' beliefs, intentions, and desires), with evidence showing that the two processes rely on partially distinct neural networks ${ }^{3}$.

White matter (WM) tracts also have a role in the ToM network. Indeed, maturity of WM connectivity was related to the emergence of mental state attribution in children ${ }^{4}$. Moreover, disconnection in WM pathways appear responsible for impaired ToM performance ${ }^{5,6}$. In patients with autism spectrum disorder (ASD), known to experience impairment in mental state attribution, WM tracts were reported to be affected ${ }^{7}$. 
The role of the cerebellum in social cognition has rarely been directly explored ${ }^{8}$. Buckner et al reported that part of the cerebellum was interconnected with the default/mentalizing network that supports ToM in human ${ }^{9}$. Moreover, connectivity between the posterior cerebellum and mentalizing areas has been reported ${ }^{10-12}$. Nevertheless, understanding the direct role of the cerebellum in ToM remains challenging. A handful of imaging studies with healthy participants demonstrated cerebellar activation when performing a mentalizing task. Yet, in a meta-analysis, only a small portion of the studies reported cerebellar activation during social judgments, including mentalizing ${ }^{13}$. Moreover, patient studies demonstrate mixed findings: while some studies show that patients with chronic cerebellar degeneration are impaired on a ToM Task ${ }^{14}$, others reported impairments but not in every patient ${ }^{15}$. In cerebellar stroke similar discrepancies were reported ${ }^{16,17}$. Moreover, studies focusing on developmental disorders characterized by a mentalizing impairment have reported cerebellar abnormalities and dysfunction of cerebellar-cortical networks ${ }^{18,19}$. Yet, those disorders are complex syndromes and also involve noncerebellar regions. Finally, there is no clear data on the lateralization of ToM processes in the cerebellum 13,20 .

To our knowledge, no studies looking at the relationship between ToM and cerebellum have been conducted using participants with focal lesions due to Traumatic Brain Injury (TBI). Moreover, no study has investigated the role of the cerebellum in the two different ToM processes (affective and cognitive). Studying participants with a focal TBI evaluated long after the trauma ensures that the primary and longlasting effect of a lesion to a particular area of the brain can be examined.

In the current study, we tested the role of the cerebellum and of the white matter (WM) tracts that support cortico-cerebellar connectivity in both cognitive and affective ToM performance and whether a laterality effect exists for the cerebellum in participants with penetrating TBI (pTBI) in participants from the Vietnam Head Injury Study (VHIS). We hypothesized that the cerebellum is part of the network that processes both (affective and cognitive) ToM processes and that disconnection of cerebello-cortical connectivity result in worse ToM performances, independently of left or right cerebellar lesion.

\section{Methods}

\subsection{Participants}

Participants were drawn from the phase 3 of the VHIS (2003-2008). This longitudinal study followed American combat veterans who suffered brain damage from pTBI in the Vietnam War, as well as neurologically healthy Vietnam combat veterans. Further details regarding the VHIS participants have previously been reported ${ }^{21}$.

Subjects were included in the present study if they completed two ToM tasks: Happe's Strange Stories Test ${ }^{22}$ and the Faux Pas Stories Test ${ }^{23}$. In total, we collected data from 193 patients with pTBI and 52 control participants. All participants understood the study procedures and gave written informed consent, 
as approved by the National Institutes of Health Neuroscience Institutional Review Board, Bethesda Naval Hospital and Department of Defense Institutional Review Boards. The Institutional Review Board at Northwestern University approved the current analysis of the data.

\subsection{Materials}

\subsubsection{Theory of mind}

While there are many tests for ToM, in this study, we focused on two that are widely used and are considered to the gold standard for assessing affective and cognitive ToM.

\subsubsection{Happe's Strange Stories Test}

Cognitive Theory of mind was measured using the Happe's Strange Stories test ${ }^{22}$. The Happe Difference Score was calculated and used as the primary outcome for the Happe's Strange Stories test. For details, see e-methods.

\subsubsection{Faux Pas Stories Test}

Affective ToM was measured using The Faux Pas test ${ }^{23}$. The Faux Pas difference score was calculated and used as the primary outcome for the Faux Pas Stories test. For details, see e-methods.

\subsubsection{Additional neuropsychological testing}

Other neuropsychological tests examined in this study included the Armed Forces Qualification Test (AFQT- 7A, 1960). Given that ToM abilities have been shown to covary with working memory ${ }^{24}$ and verbal comprehension abilities ${ }^{25}$, the WAIS Working Memory Index (WMI) and Verbal Comprehension Index (VCl) were used as covariates.

\subsection{Neuroimaging assessment and image pre-processing}

Neuroimaging assessment and image pre-processing were done using the same method described elsewhere ${ }^{26}$. Detail of the axial computed tomography (CT) acquisition is described in the e-methods. Since metal was retained in the brain due to penetrating wounds or surgical materials, MRI scans could not be acquired. Localization and analyses of the lesion were done as described in the e-methods. Figures were constructed using MRIcroGL v12.

\subsection{Statistical analyses}

\subsubsection{Voxel-based lesion-symptom mapping}

A Whole-Brain Voxel-based Lesion-Symptom Mapping (VLSM) analysis was conducted, using the same methods as described in a previous work of our group ${ }^{1}$, on the entire group of pTBI group, in order to test the association between lesion location and ToM performance on the Happe's Strange Stories test and the Faux Pas Stories test. In VLSM analyses the scores of patients with a lesion in a given voxel is 
compared to the score of patients without a lesion in this voxel using a t-test. The two primary behavioral outcomes in the VLSM analysis were the Happe Difference Score and the Faux Pas difference score. Additionally, participants' pre-injury intelligence score, education, WAIS working memory index, WAIS verbal comprehension index and lesion size were used as covariates in order to account for the possible influence of those variables. For detail, see e-methods.

\subsubsection{Lesion localization and grouping}

We then identified percent volume loss to the cerebellum for each participant in the entire pTBI group ( $\mathrm{n}=$ 193) using the automated anatomical labeling (AAL). All participants with damage to the cerebellum were selected (Cerebellar Group; $n=24)$. Note that this cerebellar group included subjects with pTBIs not restricted to the cerebellum (see Fig. 1). Participants with damage primarily to the right cerebellum ( $r$ cerebellum; $n=8$ ), left cerebellum (I cerebellum; $n=6$ ) or bilateral cerebellum (b cerebellum; $n=6$ ) were identified (see Fig. 1). Participants with a unilateral cerebellum lesion (right or left) who also had bilateral supra tentorial cortical lesions were excluded from further analysis $(n=4)$. All of the pTBI participants without a lesion in the cerebellum were selected as a control group (Other pTBIs; $n=169$, see Fig. 2 ). This group was then subdivided into patients with a unilateral left cortical lesion (I Cortical; $n=51$ ), a unilateral right cortical lesion ( $r$ Cortical; $n=65$ ) or bilateral cortical lesions (b Cortical; $n=53$ ) for additional analyses (see Fig. 2). To test the lateralization effect on the ToM tasks, both bilateral cortical and cerebellum groups were excluded. Neurologically healthy veterans also served as a comparison group (No Lesion group; $\mathrm{n}=52$ ).

\subsubsection{Behavioral data analysis}

Behavioral data analysis was carried out on both cognitive and affective ToM tasks using the same two primary behavioral outcomes of the VLSM analysis namely the Happe Difference Score (to analyze cognitive ToM) and the Faux Pas difference score (to analyze affective ToM).

We performed statistical testing using JASP $0.13 .1^{27}$ and significance level was set to $p<0.05$ (twotailed unless otherwise specified). Detail of the statistical testing is described in the e-methods.

\subsubsection{White matter tracts disconnection analysis}

To assess the degree to which specific lesions impact brain connectivity we conducted an analysis of WM disconnections contributing to ToM deficits in the cerebellar group. This was done by mapping the normalized lesion from each patient onto tractography reconstructions of WM pathways obtained from a group of healthy controls ${ }^{28}$ and quantifying the probability that the tract was disconnected by a given lesion ${ }^{29}$ using Tractotron software as part of the BCBtoolkit ${ }^{30} \mathrm{http}$ ://www.toolkit.bcblab.com]. For a similar method see ${ }^{31}$.

\section{Results}

\subsection{VLSM}




\subsubsection{Strange Stories Test}

A whole-brain VLSM analysis was performed with the Happe Difference Score as the outcome, and the following five measures as covariates: pre-injury intelligence score, WAIS working memory index score, WAIS verbal comprehension index score and lesion size. The VLSM analysis revealed three significant clusters in the cerebellum. The largest cluster (volume $=34$ voxels, Max $t=2.07$ ) was located within the left lobule VI. The peak MNI coordinates were (-30 -46 -34), and the center coordinates were (-25 -47 -30, see Fig. 3). The two other clusters were smaller (volume $=2$ voxels, Max $t=1.85$ ) and located within the left Crus I. The peak MNI coordinates were (-36 -54 -34 and - 44-56 -36 respectively), and the center coordinates were (-35 -54 -34 and $-45-55-36$ respectively; see Fig. 4a and b).

\subsubsection{Faux Pas Stories Test}

A whole-brain VLSM analysis was performed with the Faux Pas difference score as the outcome, and the following five measures as covariates: pre-injury intelligence score, WAIS working memory index score, WAIS verbal comprehension index score and lesion size. This VLSM analysis revealed no significant clusters in the cerebellum.

\subsection{Group analysis}

\subsubsection{Demographics and additional neuropsychological tests}

Demographics and neuropsychological testing results of veterans with pTBI $(n=193)$ and the No Lesion group $(n=52)$ are presented in Table 1. The following ANOVA between the cerebellar group, the other pTBI group and the No Lesion group revealed that the groups differed on the WMI score $\left(F_{(2,239)}=6.94, p\right.$ $\left.=.001 \eta^{2}=.05\right)$ with the No Lesion group scoring higher than the other $\mathrm{pTBI}$ group $\left(\mathrm{P}_{\text {bonferroni }}<.001\right)$ but not the cerebellar group ( $\left.P_{\text {bonferroni }}=.08\right)$. Nonetheless, all three groups performed within the normal range for this test. In addition, total brain volume loss did not differ among the cerebellar group and the other pTBI group $(U=2354, p=.18, R B C=.17)$. 
Table 1

Demographics and neuropsychological measures (mean (SD)) for veterans with penetrating traumatic brain injury (pTBI) and healthy controls. * Denotes significant group difference $p<0.05$.

$\begin{array}{llll}\text { Variablesigroup } & \text { PTBI } & \text { No Lesion Group Statistics } \\ & n=193 & n=52\end{array}$

Demographics:

\begin{tabular}{llll} 
Age (years) & $58.23(2.84)$ & $59.13(3.44)$ & $\mathrm{U}=4526.5, \mathrm{p}=0.35, \mathrm{RBC}=-.09$ \\
\hline Education (years) & $14.77(2.54)$ & $15.22(2.51)$ & $\mathrm{U}=4526.5, \mathrm{p}=0.35, \mathrm{RBC}=-.09$ \\
Handedness (R:L:A) ${ }^{1}$ & $160: 27: 6$ & $42: 7: 3$ & $X^{2}{ }_{(2, N=193)}=5.899, p=.21$
\end{tabular}

Neuropsychological:

\begin{tabular}{|c|c|c|c|}
\hline Pre-injury $\mathrm{IQ}^{2}$ & $60.71(25.44)$ & $68.06(21.52)$ & $U=2413.5, p=0.13, R B C=-.17$ \\
\hline Cognitive Theory of Mind ${ }^{3}$ & $0.40(3.29)$ & $-0.08(3.45)$ & $\mathrm{t}_{(243)}=.93, \mathrm{p}=0.35, \mathrm{~d}=.145$ \\
\hline Affective Theory of Mind ${ }^{4}$ & $-21.95(15.06)^{*}$ & $-16.11(11.70)^{\star}$ & $U=3816.5, p=0.08, R B C=-.24$ \\
\hline Working memory ${ }^{5}$ & $97.21(14.91)^{\star}$ & $105.71(12.57)^{\star}$ & $U=3267, p<.001, R B C=-.33$ \\
\hline Verbal comprehension ${ }^{6}$ & $106.48(15.64)$ & $110.02(11.86)$ & $U=4417, p=0.18, R B C=-.12$ \\
\hline
\end{tabular}

Handedness (L:R:A), Left, right and ambiguous.

2 Percentile score of Armed Forces Qualification Test (AFQT).

${ }^{3}$ Happe's Strange stories task: difference score between ToM and Control condition. Lower score reflects lower ToM performance.

${ }^{4}$ Faux Pas stories task: difference score between ToM and Control condition. Lower score reflects lower ToM performance.

${ }^{5}$ WAIS Working Memory Index score.

${ }^{6}$ WAIS Verbal Comprehension Index score

We next compared 4 participant groups based on lesion location: $r$ cerebellum, I cerebellum, $r$ cortical and I cortical (See Sect. 2.4.2 for grouping procedure). Demographics and neuropsychological testing results of the group analysis are shown in Table 2. No significant difference on total years of education $\left(F_{(3,126)}\right.$ $\left.=0.72, \mathrm{p}=.54, \eta^{2}=.02\right)$, pre-injury IQ scores $\left(\mathrm{F}_{(3,117)}=0.76, \mathrm{p}=.0 .52, \eta^{2}=.02\right)$, verbal comprehension score $\left(X^{2}(3, n=126)=5.83, p=.12\right)$ and working memory score were found $\left(x^{2}(3, n=125)=6.13, p=.11\right)$. Once again total brain volume loss did not differ across the 4 groups $\left(F_{(3,125)}=1.14, p=.34, \eta^{2}=.03\right)$. 
Table 2

Demographics and neuropsychological measures (mean (SD)) for veterans grouped by lesion location. * Denotes significant group difference $p<0.05$.

\begin{tabular}{|c|c|c|c|c|}
\hline Variablesigroup & $\begin{array}{l}\begin{array}{l}\text { Right } \\
\text { cerebellar }\end{array} \\
\mathrm{N}=\mathbf{8}\end{array}$ & $\begin{array}{l}\text { Left } \\
\text { cerebellar } \\
\mathrm{N}=6\end{array}$ & $\begin{array}{l}\text { Right cortex } \\
N=65\end{array}$ & $\begin{array}{l}\text { Left cortex } \\
\mathrm{N}=51\end{array}$ \\
\hline \multicolumn{5}{|l|}{ Demographics: } \\
\hline Age (years) & $58.87(1.64)$ & $58.83(0.98)$ & $58.37(2.89)$ & $58.47(2.72)$ \\
\hline Education (years) & $15.37(1.60)$ & $13.50(1.76)$ & $14.88(2.57)$ & $15.05(2.84)$ \\
\hline Handedness (R:L:A) ${ }^{1}$ & 6:2:0 & $5: 1: 0$ & $52: 10: 3$ & $45: 5: 1$ \\
\hline \multicolumn{5}{|l|}{ Neuropsychological: } \\
\hline Pre-injury $\mathrm{IQ}^{2}$ & $62.12(82.92)$ & $51.83(28.37)$ & $61.61(25.30)$ & $66.35(23.26)$ \\
\hline Cognitive Theory of Mind ${ }^{3}$ & $2.37(2.67)^{\star}$ & $-0.50(3.27)^{\star}$ & $0.03(3.29)$ & $0.88(3.57)$ \\
\hline Affective Theory of Mind ${ }^{4}$ & $-19.25(10.07)$ & $\begin{array}{l}-23.00 \\
(16.48)\end{array}$ & $-19.63(14.08)$ & $-24.14(18.67)$ \\
\hline Working memory ${ }^{5}$ & $98.00(12.22)$ & $85.50(8.48)$ & $\begin{array}{l}100.32 \\
513.33)\end{array}$ & $96.10(16.45)$ \\
\hline Verbal comprehension ${ }^{6}$ & $114.88(8.37)$ & $96.33(6.83)$ & $108.71(14.46)$ & 106.29 (19.36) \\
\hline $\begin{array}{l}\text { Total Brain Volume Loss } \\
\left(\mathrm{cc}^{3}\right)\end{array}$ & $45.79(43.45)$ & $57.62(79.22)$ & $29.24(34.02)$ & $31.89(44.22)$ \\
\hline \multicolumn{5}{|c|}{ Handedness (L:R:A), Left, right and ambiguous. } \\
\hline \multicolumn{5}{|c|}{2 Percentile score of Armed Forces Qualification Test (AFQT). } \\
\hline \multicolumn{5}{|c|}{$\begin{array}{l}{ }^{3} \text { Happe's Strange stories task: difference score between ToM and Control condition. Lower score } \\
\text { reflects lower ToM performance. }\end{array}$} \\
\hline \multicolumn{5}{|c|}{$\begin{array}{l}4 \text { Faux Pas stories task: difference score between ToM and Control condition. Lower score reflects } \\
\text { lower ToM performance. }\end{array}$} \\
\hline \multicolumn{5}{|c|}{${ }^{5}$ WAIS Working Memory Index score. } \\
\hline
\end{tabular}

\subsubsection{Theory of Mind tasks}

Regarding the ToM tasks, results of veterans with $\mathrm{pTBI}$ and the $\mathrm{HC}$ were also comparable on their performance in the Happe's Strange Stories test $\left(t_{(243)}=0.93, p=.36, d=.15\right)$ but not on their performance in the Faux Pas Stories test $(\mathrm{U}=3816.5, \mathrm{p}=.008, \mathrm{RBC}=-.24)$. The following ANOVA between the cerebellar, 
the other pTBI and the No Lesion group revealed a difference within the groups on the Faux Pas Stories Test $\left(F_{(2,242)}=3.33, p=.04 \eta^{2}=.03\right)$. However, only the difference between the No Lesion group, scoring higher, and the other pTBI group $\left(P_{\text {bonferroni }}=.03\right.$ ) survived the Bonferroni correction.

In order to confirm the lateralization of the cerebellar involvement in the ToM processes found on the VLSM analysis, we compared the performance on both ToM tasks between participants with left or right cerebellar lesions. A significant difference was found on the cognitive ToM task with participants with a left cerebellar lesion demonstrating a lower score on the Happe's Strange Stories test (one-tailed t-test; $\left.t_{(12)}=1.81, p=.05, d=.98\right)$. The mean Happe Difference score for the I cerebellar group was negative $(M=-.5, S D=3.271)$ reflecting a mean deficit in the task in the I cerebellar group (see above Sect. 2.2.1.1) whereas the mean score for the $r$ cerebellum was positive $(M=2.37, S D=2.67)$. Note here that, the mean ToM condition score for the I and $r$ cerebellar group was respectively 7 (SD: 3.2) and 11 (SD: 2.9) (max score 16) and the mean Physical condition score difference for the I and r cerebellar group was 7.5 (SD: 4.0) and 8.6 (SD: 2.4) respectively (max score 16). No norms exist for this task but the mean score of the I cerebellar group was lower than the mean score of the stroke patients (with cognitive ToM deficit) reported by Happe et al in her original publication (mean score: 10.6, SD: 3.4) ${ }^{32}$. However, no significant difference was found for the affective ToM task with no differences on the Faux Pas Stories test between the groups $\left(t_{(12)}=.53, p=.61, d=.29\right)$.

Next, we conducted linear regression analyses to test whether damage to left Crus I and lobule VI was specifically associated with cognitive ToM performance. The regression model included the Happe differences score as the dependent variable, and the following as covariates: pre-injury intelligence score, years of formal education, WAIS working memory index score, WAIS verbal comprehension index score, percent damage to left Crus I and lobule VI and the total volume loss. Overall, the model explained a significant proportion of variance in cognitive ToM performance $\left(R^{2}=0.79, F_{(7,14)}=3.76, p=.05\right)$, with more damage to the left Crus I predicting lower ToM performance ( $\beta=0.73 ; t=3.06, p=.02$,one-tailed) as well as more damage to the left lobule VI ( $\beta=-0.67 ; t=-3.32, p=.01$, one-tailed). No other covariate contributed significantly to the model including right cerebellar structures.

The same linear regression analyses were conducted for the performance on affective ToM. None of the covariates contributed significantly to the model including the right or left cerebellar structures.

\subsection{White matter tracts disconnection}

Cerebellar group participants' lesions were compared to an atlas of WM connections in order to identify the probability of tract disconnections ${ }^{28,30}$. The percentage of patients with disconnected tracts was calculated separately only for patients with and without deficits in cognitive ToM (determined based on zero as a cut-off score, difference score equal or higher than 0 reflects no deficit, score $<0$ reflects deficit), for patients with a left cerebellar lesion $(n=6)$ and a right cerebellar lesion $(n=8)$. Only cognitive ToM was explored as no significant results were found either on the VLSM or on group analysis for the affective ToM task. We compared the groups of patients with and without presumed damage for each 
WM tract separately, using a chi-square test. This analysis revealed that disconnections of the left Thalamic projection $\left(X^{2}(1, N=14)=4.20 p=.04\right)$, and the left Fronto-Striatal fasciculus $\left(X^{2}(1, N=14)=\right.$ $4.20 p=.04$ ) were modestly associated with a poorer performance in the cognitive ToM task. However, these comparisons did not survive Bonferroni correction for multiple comparisons.

\section{Discussion}

In this study we explored the role of the cerebellum in ToM. We found that a lesion to the left cerebellum, and more specifically to the left Crus I and lobule VI, led to a deficit in cognitive ToM but not affective ToM. We also found that disconnection of the cerebello-cortical pathways through the left fronto-striatal tract and the left thalamic projection were more likely to result in cognitive ToM deficits.

Overall, these findings suggest that the left cerebellum contributes to the cognitive processes of mental state attribution.

\section{The difference between cognitive and affective Theory of Mind}

To our knowledge, our study is the first to report that the cerebellum is an important node in the Cognitive but not in the Affective ToM network. This is in line with previous findings suggesting a distinct neural network for each ToM process ${ }^{33}$. Our study adds a novel region to these networks.

\section{The role of the cerebellum in Theory of Mind neural network}

The role of the cerebellum in social cognition emerged in the last decade. In a first meta-analysis, Van Overwall et a/ reported that cerebellar "social" clusters overlapped with nonsocial function clusters ${ }^{13}$. Therefore, they argued that the cerebellum provides a domain-general support of social cognition ${ }^{13}$. However, after reporting that their mentalizing clusters were located mostly within the default network ${ }^{9}$, they concluded that the cerebellum provides a domain-specific process for social cognition ${ }^{10}$.

Regarding the anatomical localization of cerebellar regions that are associated with mentalism, we found a ToM cluster only for the cognitive task, and only in the left cerebellar hemisphere. Our clusters were more lateral than previously described. When compared to the ToM activation map of King et al, our clusters are outside the ToM map and are all within region 6 (Active maintenance, divided attention, verbal fluency) ${ }^{20}$. Also, when compared to the Buckner et al 7 network map, our clusters are not within the default network but within the ventral attention network and the executive/cognitive network ${ }^{9}$. These differences may be related to the difference in the subject sample, the tasks used and our analytic approach. However, even in the previous studies, there were inconsistencies regarding which cerebellar lobules were involved and if a laterality effect existed. Van Overwall et a/ reported that person mentalizing 
clusters were associated with the right Crus I, left and right lobule $\mathrm{VI}$ and right lobule IV ${ }^{13}$. But when using another type of analysis, the clusters were encompassed in left and right Crus ${ }^{8}{ }^{8}$. Moreover, King et al reported that most of the ToM activations were within left and right Crus I and II (with a larger lateral spread on the right side) but with extension to right lobule VI and IX and to the midline (vermis) ${ }^{20}$. However, in a recent functional neuroimaging study, it was reported that impairment on a complex ToM task was associated with decreased volume of the left lobule $\mathrm{VI}^{34}$. Our results corroborate these latter findings.

\section{The role of the cerebellum in cognitive Theory of Mind}

It is widely accepted that the cerebellum has a role in predicting motor outcome and signaling the cortex when errors are detected, in order to update the motor signal and reduce errors in future movements ${ }^{35}$. Similarly to motor activity, social behavior also require anticipation and adaptation ${ }^{36,37}$. Motor and space processing may help characterize environmental constraints on social behavior by processing of physical boundaries, agent sequences, and coordination. In particular, adaptation and prediction of the behavior of the self and others could be a very specific contribution that the Cerebellum makes to understanding the intent of others ${ }^{14,38}$. This idea supports previous arguments claiming that the cerebellum regulates cortical functions for complex social behaviors by enhancing the feedforward control that is necessary to perform these functions correctly ${ }^{39,40}$. One hypothesis why the cerebellum may be involved in cognitive but not affective ToM results follows directly from this interpretation. Cognitive ToM is a more complex form of ToM compared to affective ToM because it subserves higherorder cognitive and metacognitive processes ${ }^{34}$. Affective ToM tasks would require less prediction and therefore, would not tax the cerebellum as much as cognitive ToM. The Clausi et al findings support this by reporting no impairment in a task that assesses the ability to attribute emotions to others in a social context and argue that it was because the task requires reduced prediction and interplay between cognitive and emotional aspects ${ }^{41}$.

Another hypothesis is that the role of the cerebellum in the cognitive ToM task is in controlling the sensorimotor aspects of ToM. Indeed, it was reported that Cognitive but not affective ToM deficit was also related to working memory performance ${ }^{42}$. Our group has shown that the role of the cerebellum in executive function is supportive since it appears to primarily compute the motor component of working memory ${ }^{26}$. Also, in patients with cervical dystonia, it was reported that only cognitive ToM was impaired in those with tremor compared to those without motor impairment ${ }^{43}$. Therefore, one could hypothesize that cognitive ToM tasks rely more upon sensorimotor control than affective ToM tasks.

\section{The role of the white matter tracts}

The cerebellum is interconnected with the cerebrum via cerebello-cortical WM loops. In our study, the WM disconnection analysis revealed that damage to the WM pathways that include the "relay" structures of 
the cerebello-cortical WM connection, namely the thalamus and the striatum, were associated with poor performance in cognitive ToM. This finding suggests an important role for the cerebellum in the neural network that supports cognitive ToM and that integrity of the cerebello-cortical tracts is essential for cognitive ToM.

\section{Limitations}

All participants were male veterans and mostly Caucasian, therefore this limit our ability to generalize the results to other populations. Moreover, there are documented sex differences in ToM ${ }^{44}$ which could not be addressed. As happens in longitudinal studies, participants in the current phase of the study are likely to have recovered better from their injury than patients who would be assessed shortly after their injury. Yet, our ability to identify impairments in this set of patients suggest that cerebellar damage can lead to poorer ToM ability even after several decades. Finally, as noted in the methods section, only CT images were used, but MRI and DTI tractography should also be used for white matter pathways identification in future studies with other patient populations.

\section{Conclusions}

This study is the first to provide direct causal neuropsychological evidence for an important role of the cerebellum in cognitive ToM processing but not in affective ToM. Our results indicate that human social cognition relies on a complex network functionally connected through WM pathways that include the cerebellum. It provides evidence that it is the left cerebellar Crus I and lobule VI that contributes to cognitive mental attribution. It also supports evidence that the neural networks underpinning cognitive and affective ToM can be differentiated.

\section{Declarations}

\section{Fundings:}

This research was supported by the Therapeutic Cognitive Neuroscience Fund (B. Gordon).

\section{Acknowledgments:}

The First Author (PAB) would like to gratefully thank the "Gueules Cassées" Foundation, the Servier Institute, the Philippe Foundation, the "Hospices Civils de Lyon", the French Society of Neurosurgery and the French Society of Pediatric Neurosurgery for their support. The authors would like to thank all the Vietnam veterans who participated in this study. Without their long-term commitment to improving the health care of veterans, this study could not have been completed. We also thank J. Solomon as well as AM. Salazar, V. Raymont, S. Bonifant, B. Cheon, C. Ngo, A. Greathouse, K. Reding, and G. Tasick for testing and evaluating participants. We would also like to thank the National Naval Medical Center and the 
National Institute of Neurological Disorders and Stroke for providing support and facilities to conduct this study. This effort was supported in part by Johns Hopkins' Therapeutic Cognitive Neuroscience Fund (BG). The views expressed in this article are those of the authors and do not reflect the official policy or position of the Department of the Navy, the Department of Defense, or the U.S. Government.

\section{Disclosures:}

Pierre-Aurélien BEURIAT reports no disclosures relevant to this manuscript

Shira COHEN-ZIMERMAN reports no disclosures relevant to this manuscript

Gretchen N.L SMITH reports no disclosures relevant to this manuscript

Frank KRUEGER reports no disclosures relevant to this manuscript

Barry GORDON reports no disclosures relevant to this manuscript

Jordan GRAFMAN reports no disclosures relevant to this manuscript

\section{DATA AVAILABILITY STATEMENT}

The data that support the findings of this study are available from the corresponding author upon reasonable request. This study was not preregistered.

\section{CONTRIBUTIONS}




\begin{tabular}{|lll|}
\hline Name & Location & Contribution \\
\hline $\begin{array}{l}\text { Pierre- } \\
\text { Aurélien } \\
\text { BEURIAT, MD, } \\
\text { PhD }\end{array}$ & $\begin{array}{l}\text { Shirley Ryan AbilityLab, } \\
\text { Chicago }\end{array}$ & $\begin{array}{l}\text { designed research; analyzed and interpreted the data; } \\
\text { wrote the paper }\end{array}$ \\
\hline $\begin{array}{l}\text { Shira } \\
\text { COHEN- } \\
\text { ZIMERMAN, } \\
\text { PhD }\end{array}$ & $\begin{array}{l}\text { Shirley Ryan AbilityLab, } \\
\text { Chicago }\end{array}$ & $\begin{array}{l}\text { designed research; analyzed and interpreted the data; } \\
\text { revised the manuscript }\end{array}$ \\
\hline $\begin{array}{l}\text { Gretchen N.L } \\
\text { SMITH, PhD }\end{array}$ & $\begin{array}{l}\text { Shirley Ryan AbilityLab, } \\
\text { Chicago }\end{array}$ & revised the manuscript \\
$\begin{array}{l}\text { Frank } \\
\text { KRUEGER, } \\
\text { PhD }\end{array}$ & $\begin{array}{l}\text { George Mason } \\
\text { University, Fairfax }\end{array}$ & $\begin{array}{l}\text { designed and performed research; revised the } \\
\text { manuscript }\end{array}$ \\
\hline $\begin{array}{l}\text { Barry } \\
\text { GORDON, } \\
\text { MD }\end{array}$ & $\begin{array}{l}\text { Johns Hopkins } \\
\text { University School of } \\
\text { Medicine, Baltimore }\end{array}$ & $\begin{array}{l}\text { designed and performed research; revised the } \\
\text { manuscript }\end{array}$ \\
$\begin{array}{l}\text { Jordan } \\
\text { GRAFMAN, } \\
\text { PhD }\end{array}$ & $\begin{array}{l}\text { Shirley Ryan AbilityLab, } \\
\text { Chicago }\end{array}$ & $\begin{array}{l}\text { designed and performed research; analyzed and } \\
\text { interpreted the data; acquired the data; wrote the paper; } \\
\text { supervised the study }\end{array}$ \\
\hline
\end{tabular}

\section{References}

1. Cohen-Zimerman, S. et al. The neural basis for mental state attribution: A voxel-based lesion mapping study. Hum. Brain Mapp, https://doi.org/10.1002/hbm.25203 (2020).

2. Baldo, J. V., Kacinik, N. A., Moncrief, A., Beghin, F. \& Dronkers, N. F. You may now kiss the bride: Interpretation of social situations by individuals with right or left hemisphere injury., 80, 133-141 (2016).

3. Shamay-Tsoory, S. G., Tibi-Elhanany, Y. \& Aharon-Peretz, J. The ventromedial prefrontal cortex is involved in understanding affective but not cognitive theory of mind stories. Soc. Neurosci, 1, 149166 (2006).

4. Grosse Wiesmann, C., Schreiber, J., Singer, T., Steinbeis, N. \& Friederici, A. D. White matter maturation is associated with the emergence of Theory of Mind in early childhood. Nat. Commun, 8, 14692 (2017).

5. Batista, S. et al. Disconnection as a mechanism for social cognition impairment in multiple sclerosis. Neurology, 89, 38-45 (2017).

6. Downey, L. E. et al. White matter tract signatures of impaired social cognition in frontotemporal lobar degeneration. Neurolmage Clin, 8, 640-651 (2015).

7. Bradstreet, L. E., Hecht, E. E., King, T. Z., Turner, J. L. \& Robins, D. L. Associations between autistic traits and fractional anisotropy values in white matter tracts in a nonclinical sample of young adults. Exp. Brain Res, 235, 259-267 (2017). 
8. Van Overwalle, F. et al. Consensus Paper: Cerebellum and Social Cognition. Cerebellum Lond. Engl, https://doi.org/10.1007/s12311-020-01155-1 (2020).

9. Buckner, R. L., Krienen, F. M., Castellanos, A., Diaz, J. C. \& Yeo, B. T. T. The organization of the human cerebellum estimated by intrinsic functional connectivity. J. Neurophysiol, 106, 2322-2345 (2011).

10. Van Overwalle, F., D'aes, T. \& Mariën, P. Social cognition and the cerebellum: A meta-analytic connectivity analysis. Hum. Brain Mapp, 36, 5137-5154 (2015).

11. Van Overwalle, F. \& Mariën, P. Functional connectivity between the cerebrum and cerebellum in social cognition: A multi-study analysis. Neurolmage, 124, 248-255 (2016).

12. Van Overwalle, F., Van de Steen, F. \& Mariën, P. Dynamic causal modeling of the effective connectivity between the cerebrum and cerebellum in social mentalizing across five studies. Cogn. Affect. Behav. Neurosci, 19, 211-223 (2019).

13. Van Overwalle, F., Baetens, K., Mariën, P. \& Vandekerckhove, M. Social cognition and the cerebellum: A meta-analysis of over 350 fMRI studies. Neurolmage, 86, 554-572 (2014).

14. Sokolov, A. A. The Cerebellum in Social Cognition.Front. Cell. Neurosci.12, (2018).

15. Sokolovsky, N., Cook, A., Hunt, H., Giunti, P. \& Cipolotti, L. A preliminary characterisation of cognition and social cognition in spinocerebellar ataxia types 2, 1, and 7. Behav. Neurol, 23, 17-29 (2010).

16. Roldan Gerschcovich, E., Cerquetti, D., Tenca, E. \& Leiguarda, R. The impact of bilateral cerebellar damage on theory of mind, empathy and decision making. Neurocase, 17, 270-275 (2011).

17. Roca, M., Gleichgerrcht, E., Ibáñez, A., Torralva, T. \& Manes, F. Cerebellar stroke impairs executive functions but not theory of mind. J. Neuropsychiatry Clin. Neurosci, 25, E48-49 (2013).

18. Andreasen, N. C., Calarge, C. A., Calage, C. A. \& O'Leary, D. S. Theory of mind and schizophrenia: a positron emission tomography study of medication-free patients. Schizophr. Bull, 34, 708-719 (2008).

19. Fatemi, S. H. et al. Consensus paper: pathological role of the cerebellum in autism. Cerebellum Lond. Engl, 11, 777-807 (2012).

20. King, M., Hernandez-Castillo, C. R., Poldrack, R. A., Ivry, R. B. \& Diedrichsen, J. Functional boundaries in the human cerebellum revealed by a multi-domain task battery. Nat. Neurosci, 22, 1371-1378 (2019).

21. Barbey, A. K. et al. An integrative architecture for general intelligence and executive function revealed by lesion mapping. Brain J. Neurol, 135, 1154-1164 (2012).

22. Happé, F. G. E. An advanced test of theory of mind: Understanding of story characters' thoughts and feelings by able autistic, mentally handicapped, and normal children and adults. J. Autism Dev. Disord, 24, 129-154 (1994).

23. Stone, V. E., Baron-Cohen, S. \& Knight, R. T. Frontal lobe contributions to theory of mind. J. Cogn. Neurosci, 10, 640-656 (1998).

24. Gokcen, S., Bora, E., Erermis, S., Kesikci, H. \& Aydin, C. Theory of mind and verbal working memory deficits in parents of autistic children. Psychiatry Res, 166, 46-53 (2009). 
25. White, S., Hill, E., Happé, F. \& Frith, U. Revisiting the strange stories: Revealing mentalizing impairments in autism. Child Dev, 80, 1097-1117 (2009).

26. Beuriat, P. A. et al. A New Insight on the Role of the Cerebellum for Executive Functions and Emotion Processing in Adults. Front. Neurol.11, (2020).

27. JASPTeam. JASP (Version 0.12.2). (2020).

28. Rojkova, K. et al. Atlasing the frontal lobe connections and their variability due to age and education: a spherical deconvolution tractography study. Brain Struct. Funct, 221, 1751-1766 (2016).

29. de Thiebaut, M. et al. Damage to white matter pathways in subacute and chronic spatial neglect: a group study and 2 single-case studies with complete virtual "in vivo" tractography dissection. Cereb. Cortex, 24, 691-706 (2014).

30. Foulon, C. et al. Advanced lesion symptom mapping analyses and implementation as BCBtoolkit. Gigascience, 7, giy004 (2018).

31. Chechlacz, M., Rotshtein, P. \& Humphreys, G. W. Neuronal substrates of Corsi Block span: Lesion symptom mapping analyses in relation to attentional competition and spatial bias., $64,240-251$ (2014).

32. Happé, F., Brownell, H. \& Winner, E. Acquired 'theory of mind' impairments following stroke., 70, 211240 (1999).

33. Abu-Akel, A. \& Shamay-Tsoory, S. Neuroanatomical and neurochemical bases of theory of mind., 49, 2971-2984 (2011).

34. Quidé, Y., Wilhelmi, C. \& Green, M. J. Structural brain morphometry associated with theory of mind in bipolar disorder and schizophrenia. PsyCh J, 9, 234-246 (2020).

35. Ito, M. Cerebellar circuitry as a neuronal machine. Prog. Neurobiol, 78, 272-303 (2006).

36. Sokolov, A. A., Miall, R. C. \& Ivry, R. B. The Cerebellum: Adaptive Prediction for Movement and Cognition. Trends Cogn. Sci, 21, 313-332 (2017).

37. Brown, E. C. \& Brüne, M. The role of prediction in social neuroscience. Front. Hum. Neurosci, 6, 147 (2012).

38. Ito, M. Opinion - Control of mental activities by internal models in the cerebellum. Nat. Rev. Neurosci, 9, 304-313 (2008).

39. Leggio, M. G., Chiricozzi, F. R., Clausi, S., Tedesco, A. M. \& Molinari, M. The neuropsychological profile of cerebellar damage: The sequencing hypothesis. Cortex J. Devoted Study Nerv. Syst. Behav, 47, 137-144 (2011).

40. Leggio, M. \& Molinari, M. Cerebellar sequencing: a trick for predicting the future. Cerebellum Lond. Engl, 14, 35-38 (2015).

41. Clausi, S. et al. The Cerebellar Predictions for Social Interactions: Theory of Mind Abilities in Patients With Degenerative Cerebellar Atrophy. Front. Cell. Neurosci, 12, 510 (2018).

42. Bottiroli, S., Cavallini, E., Ceccato, I., Vecchi, T. \& Lecce, S. Theory of Mind in aging: Comparing cognitive and affective components in the faux pas test. Arch. Gerontol. Geriatr, 62, 152-162 (2016). 
43. Lagravinese, G. et al. Affective and cognitive theory of mind in patients with cervical dystonia with and without tremor. J. Neural Transm. Vienna Austria, 1996, https://doi.org/10.1007/s00702-02002237-4 (2020).

44. Adenzato, M. et al. Gender differences in cognitive Theory of Mind revealed by transcranial direct current stimulation on medial prefrontal cortex. Sci. Rep, 7, 41219 (2017).

\section{Figures}




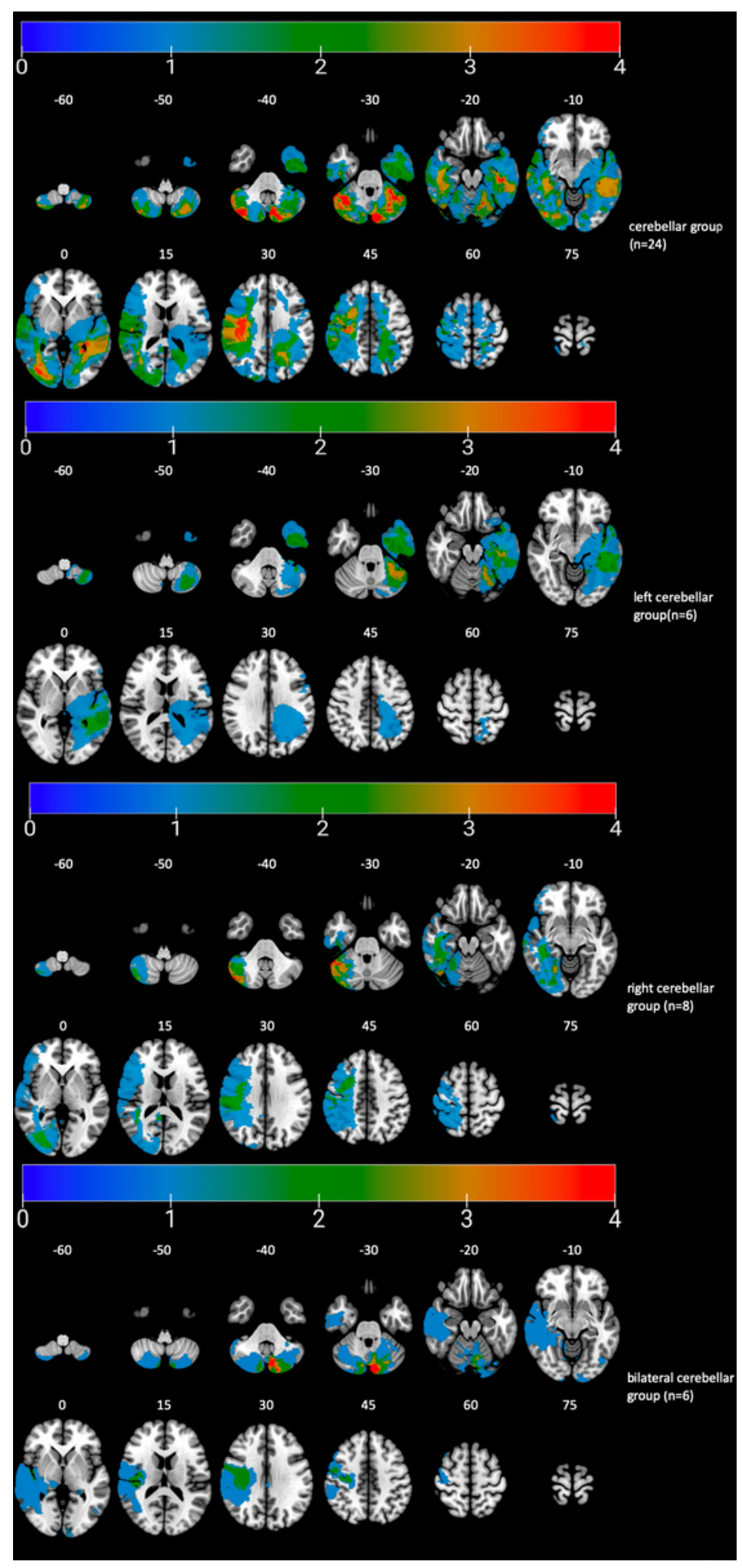

\section{Figure 1}

lesion overlay maps of participant with cerebellar lesion $(n=24)$ grouped by lesion location. Numbers on the top of the brain slices indicate the $z$ coordinates (MNI) of each axial slice. The color indicates the number of veterans in the group with damage to a given voxel. Images are in radiological space (i.e. right is left). For interpretation of the references to color in this figure legend, the reader is referred to the web version of this article 


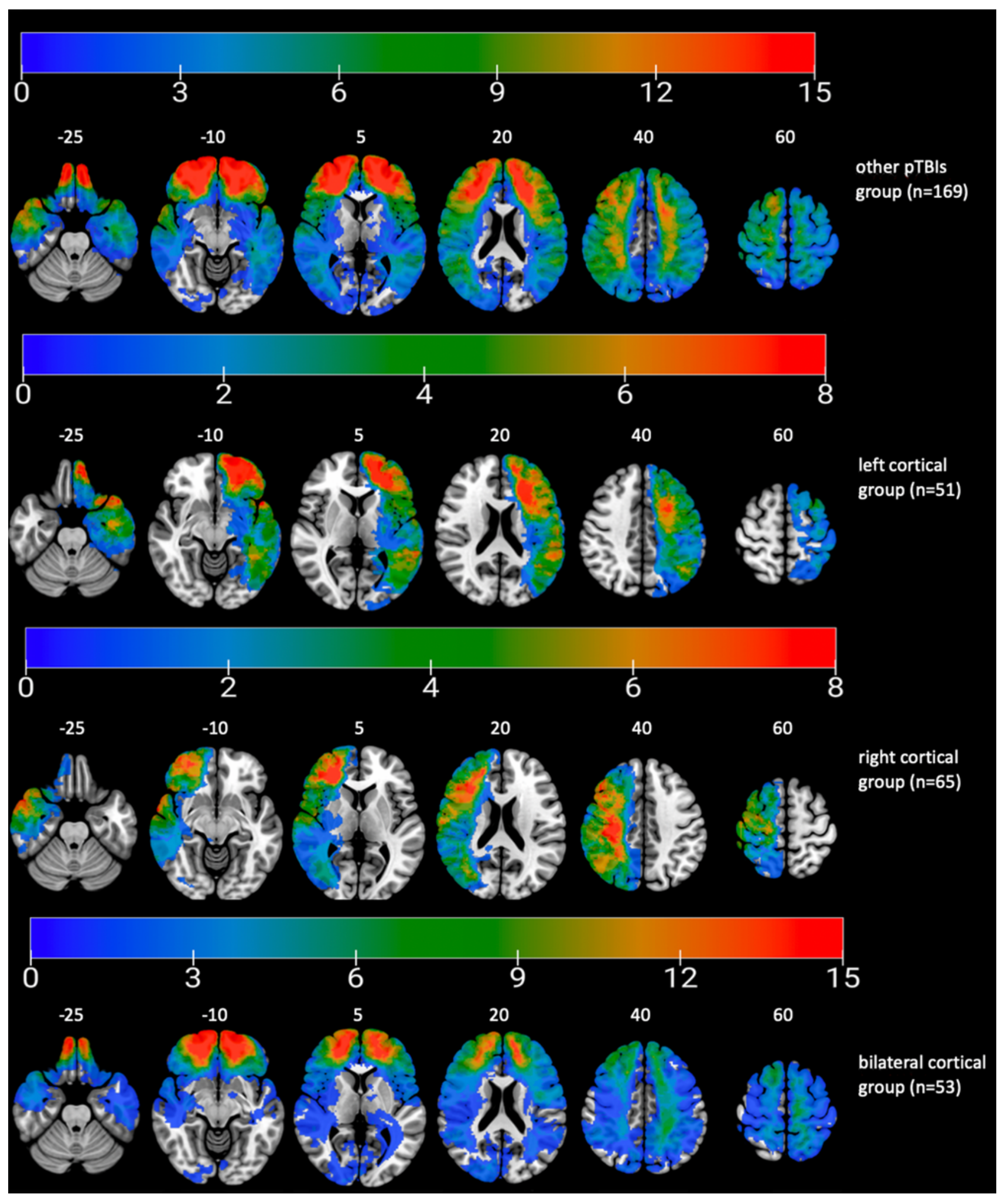

Figure 2

lesion overlay maps of participant in the other pTBIs group $(n=169)$ grouped by lesion location. Numbers on the top of the brain slices indicate the $z$ coordinates $(\mathrm{MNI})$ of each axial slice. The color indicates the number of veterans in the group with damage to a given voxel. Images are in radiological space (i.e. right is left). For interpretation of the references to color in this figure legend, the reader is referred to the web version of this article 

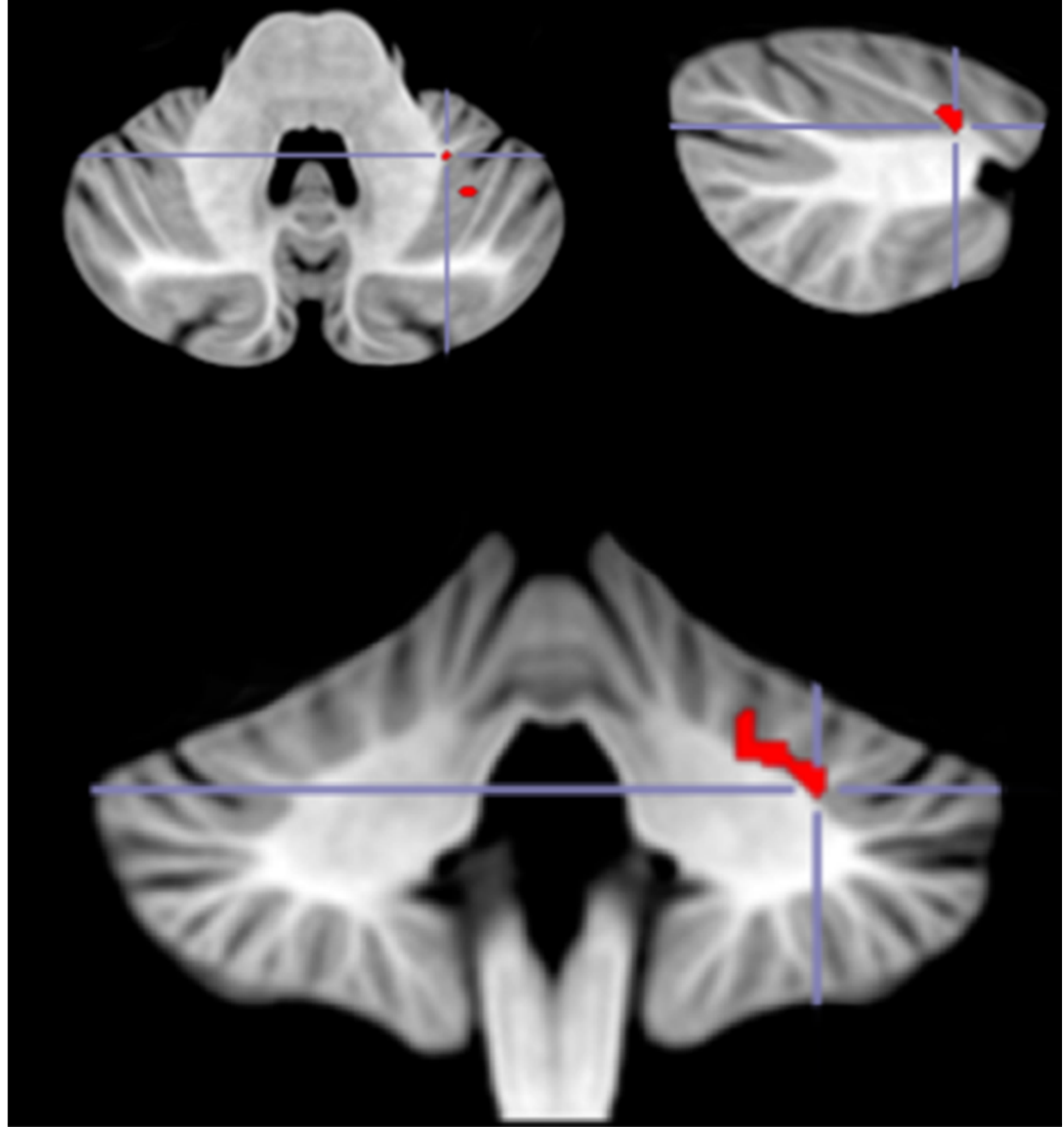

\section{Figure 3}

VLSM analysis results. In red are areas of damage in the left lobule VI that were associated with a deficit in cognitive ToM. Peak MNI coordinates for the main cluster (-30 -46-34). 

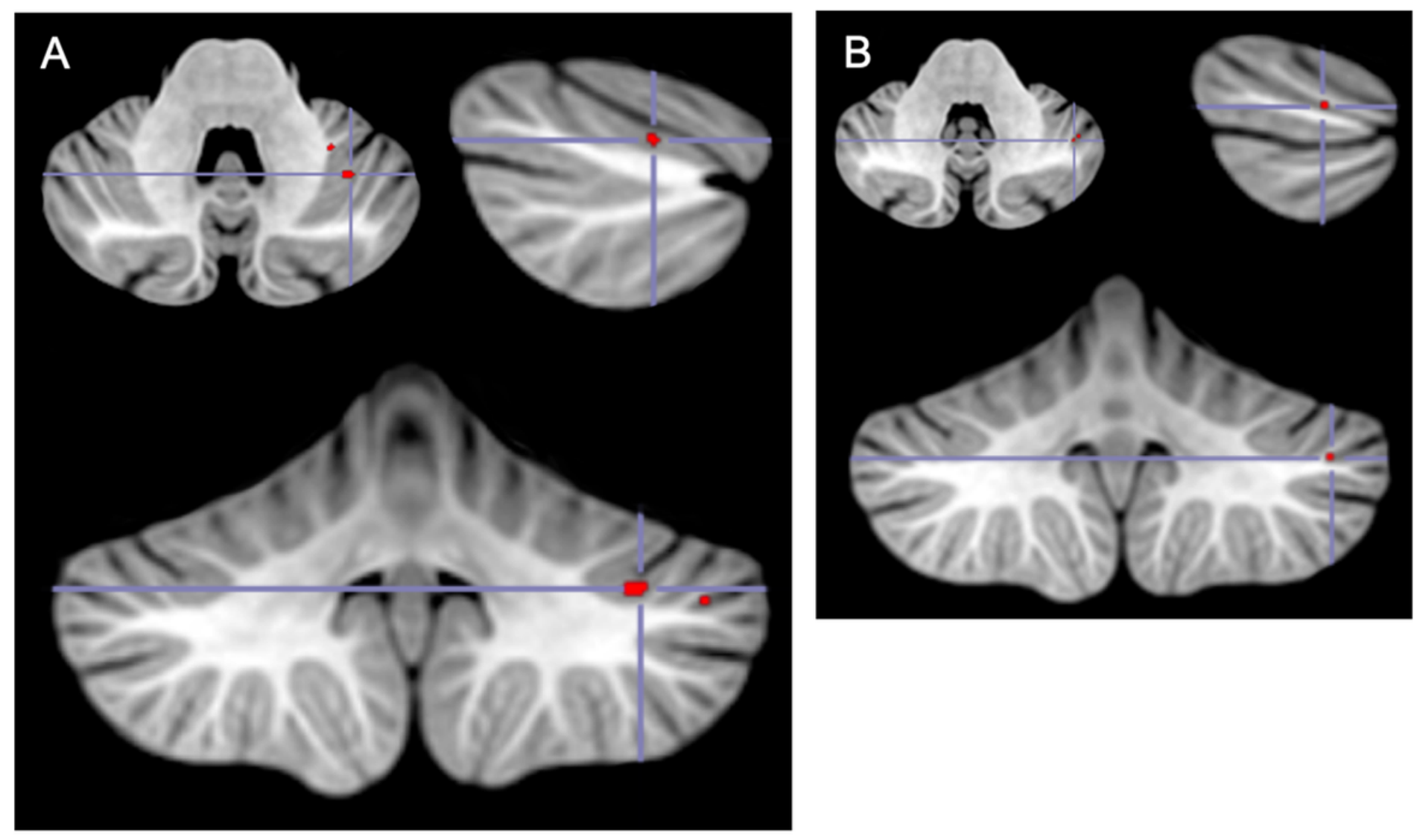

\section{Figure 4}

A and B: VLSM analysis results. In red are areas of damage in the left Crus I that were associated with a deficit in cognitive ToM. Peak MNI coordinates for the two main cluster (A (-36 -54 -34) and B (-44 -56 -36)).

\section{Supplementary Files}

This is a list of supplementary files associated with this preprint. Click to download.

- SupplementarySR.docx 